\title{
LETTER TO THE EDITOR MicroRNA-155 regulates casein kinase 1 gamma 2: a potential pathogenetic role in chronic lymphocytic leukemia
}

Blood Cancer Journal (2017) 7, e606; doi:10.1038/bcj.2017.80; published online 8 September 2017

Chronic lymphocytic leukemia (CLL) is the most common leukemia of adults in Western countries. There are approximately 19000 new cases diagnosed in the United States in 2016 (https://seer. cancer.gov/statfacts/html/clyl.html). MicroRNAs (miRs) have been implicated as one of the key contributors in the pathogenesis of CLL. One of these miRNAs is miR-155, which is probably the bestcharacterized miRNA involved in B-cell maturation and function. MiR-155 has a critical role in normal immune function, ${ }^{1}$ including innate response, regulation of the germinal center response and formation of class-switched plasma cells and negative regulation of activation-induced cytidine deaminase. However, miR-155 is also an oncogenic miRNA overexpression of miR-155 in mice or its precursor $B I C$ in chickens led to the development of lymphomas. Furthermore, abnormal expression of miR-155 has been observed in a number of lymphoid malignancies, including diffuse large B-cell lymphoma, classical Hodgkin lymphoma, primary mediastinal B-cell lymphoma and CLL. ${ }^{2}$ In various studies, miR-155 has been found consistently overexpressed in CLL cells compared with normal B cells. Although there does not appear to be definitive correlations between miR-155 expression levels and individual CLL prognostic factors, high pretreatment levels of miR-155 in CLL cells or in plasma have been demonstrated to be associated with shorter need-for-treatment interval and failure to achieve complete response, respectively. ${ }^{3,4}$ The association of adverse clinical outcome in CLL with high miR-155 levels appears to be linked to the capacity of miR-155 to enhance the sensitivity of CLL cells to B-cell receptor ligation. ${ }^{5}$ Thus it is likely that miR-155 has important roles in the pathobiology of CLL.

Identification of targets for miR-155 will facilitate the understanding of how its deregulation contributes to the pathogenesis of CLL. To identify physiologically relevant targets of miR-155 in CLL, we measured miR-155 levels of purified CLL B cells from the peripheral blood of 38 patients using quantitative reverse transcriptase-PCR, as well as 5 sets of naive B cells and 6 sets of memory $B$ cells isolated from hyperplastic tonsils. The results were ranked according to miR-155 levels and IGVH status (Supplementary Figure S1). MiR-155 levels in CLL with high miR-155 expression (11 in total) are about fivefold that of CLL with low miR-155 expression (11 in total). The six top-ranked and five bottom-ranked CLLs in each of the IGVH-unmutated and -mutated subgroups were selected based on miR-155 expression for global cDNA expression microarray analysis (supplementary Table S1) using Affymetrix HG-U133 Plus 2.0 microarrays (Cat no. 900466, Affymetrix Inc., Santa Clara, CA, USA) containing $>54000$ probe sets covering $>20000$ characterized human genes. A comparison between the expression profiles of CLL with high miR-155 vs low miR-155 using significance analysis of microarray analysis (5\% false discovery rate) revealed 10 probe sets derived from 8 genes that are downregulated (Supplementary Table S2). Three of these genes: CSNK1G2, ZNF652, and KLF3, harbor evolutionarily conserved miR-155 binding sites according to prediction by TargetScan (v 7.1, June 2016). To further validate CSNK1G2 as a miR-155 target, we generated luciferase reporter plasmids with human CSNK1G2 3'-untranslated region containing either wildtype or mutated miR-155-binding site to perform luciferase reporter assays. The results confirmed that miR-155 could repress the reporter gene expression in 293 T cells and that this repressive effect could be relieved by mutation in the seed region of the miR-155-binding site (Supplementary Figure S2).

Within the original cohort, CLLs with low miR-155 had significantly higher levels ( 1.6-fold) of CSNK1G2 mRNA compared with CLLs with high miR-155 (Figure 1a), which validates the results of microarray analysis and is consistent with a negative regulatory effect of miR-155 on CSNK1G2 mRNA levels. For additional validation, a cohort of 43 additional CLL samples were tested Supplementary Table S3. We did not detect any significant correlation between the miR-155 levels and CSNK1G2 mRNA expression levels for the new cohort when the entire cohort was analyzed (Figure 1b). When CSNK1G2 mRNA expression levels were compared between the subgroup of six patients with the highest miR-155 levels and the subgroup of six patients with the lowest miR-155 levels, the high miR-155 subgroup showed a trend of lower CSNK1G2 levels (Figure 1c). The difference was not statistically significant $(P=0.17)$. However, when the cohort was subdivided into IGVH-unmutated and -mutated groups, we found a moderate correlation $\left(R^{2}=0.42\right)$ between miR-155 and CSNK1G2 mRNA levels for the IGVH-mutated group but not for the IGVHunmutated group, and the CSNK1G2 mRNA expression was significantly higher for the low miR-155 expression in the IGVHmutated subgroup $(P=0.04$, Figures $1 \mathrm{~d}-\mathrm{g}$ ). We were unable to determine whether similar negative correlation can be seen between miR-155 and CSNK1G2 protein, as western blotting of protein extracts isolated form primary CLL cells using commercially available antibodies has been unsuccessful in detecting specific bands despite repeated attempts.

We also enforced miR-155 expression in CLL cells to determine its effect on CSNK1G2 expression. MiR-155 mimic (Cat. no. 472490001, Exiqon, Vedbæk, Denmark) was introduced into fresh purified CLL B cells from the peripheral blood of patients with IGVH mutation and relatively low miR-155 levels. A 1.6-fold Increase in miR-155 resulted in reduction of CSNK1G2 and SOCS1 mRNA (as positive control) levels by $17 \%$ and $31 \%$, respectively, compared with CLL cells transfected with negative control microRNA (miRCURY LNA microRNA mimic Negative Control 4, Exiqon, Cat. no. 479903-001) (Figures 2a and b). These findings provided direct experimental evidence of a role of miR-155 in inhibiting CSNK1G2 expression, particularly in IGVH-mutated CLL cells.

Our findings suggest that CSNK1G2 is a physiologically relevant miR-155 target in CLL cells. Previously, CSNK1G2 has been predicted to be a miR-155 target in diffuse large B-cell lymphoma based on transcriptome profiling ${ }^{6}$ and also by Argonaute-2 RNA immunoprecipitation followed by next-generation sequencing in 293 T cells with enforced miR-155 expression. ${ }^{7}$ CSNK1G2 is an isoform of casein kinase I, an evolutionarily conserved family of serine/threonine kinases implicated in multiple cellular functions, such as differentiation, proliferation and chromosomal segregation. One of the targets of CSNK1G2 is the collagen type IV alpha 3 binding protein (COL4A3BP/CERT), phosphorylation of which can lead to its inactivation and consequent downregulation of endoplasmic reticulum-to-Golgi transport of ceramide and 

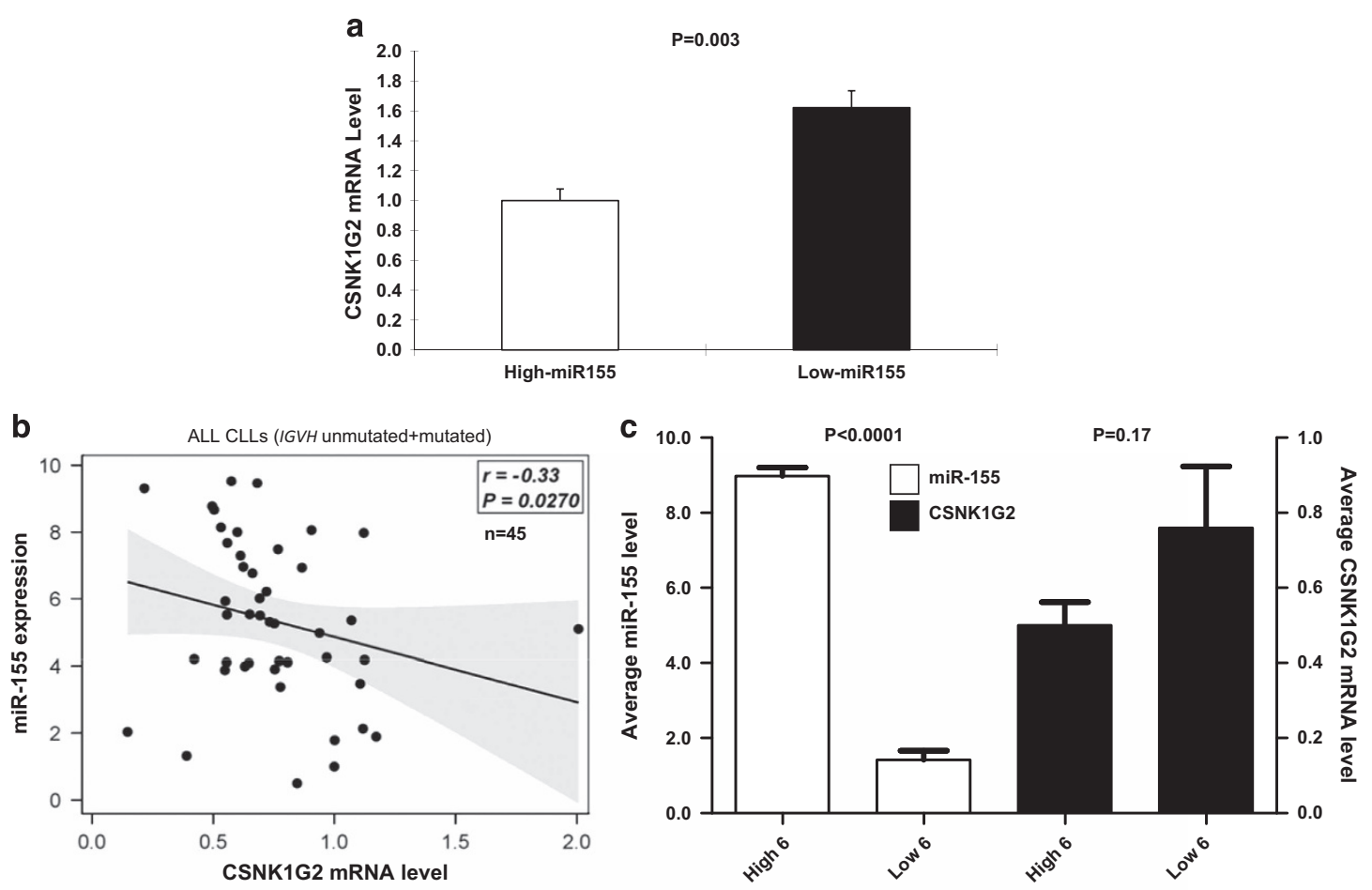

d

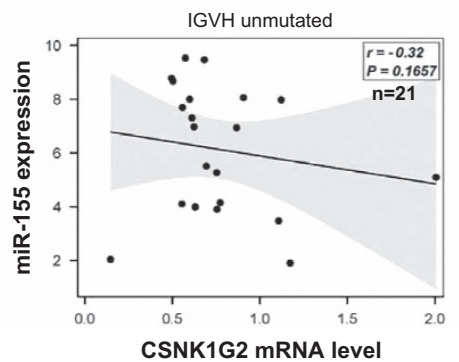

e
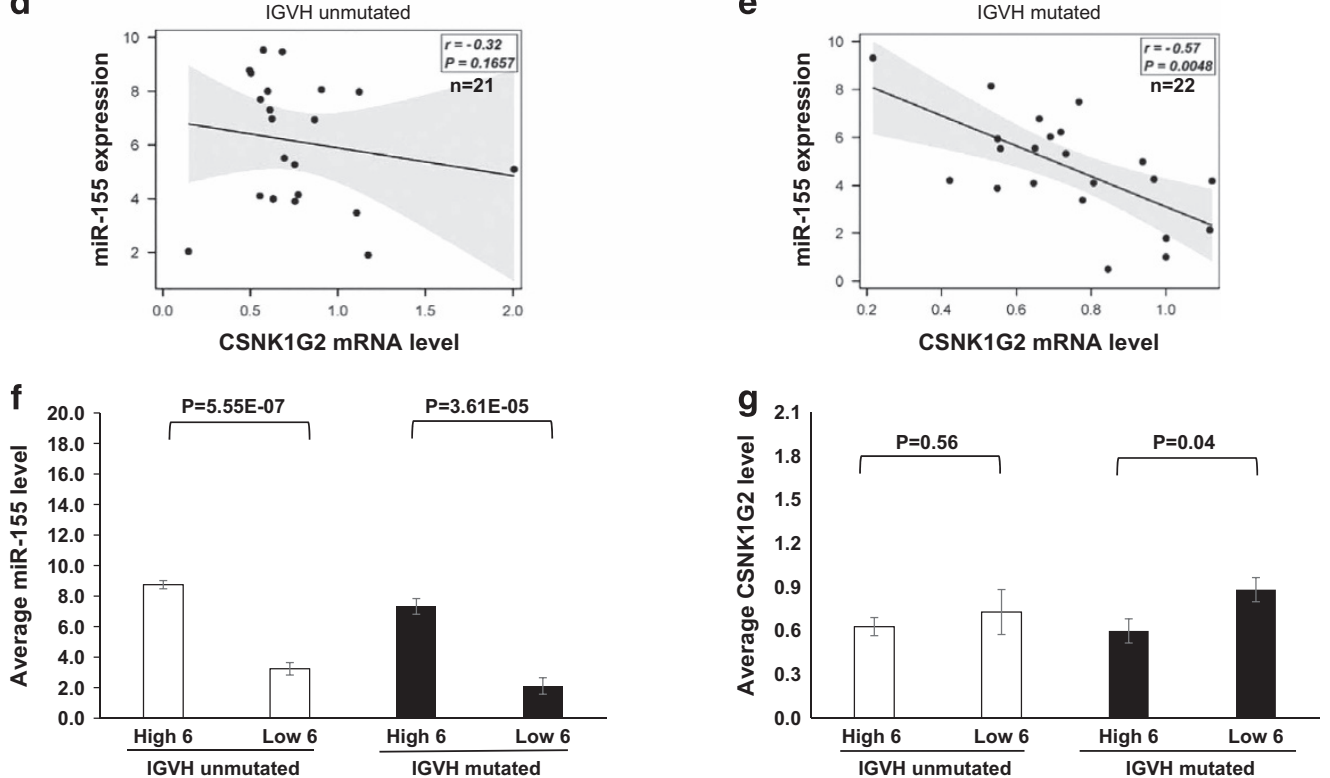

Figure 1. Expression correlation between hsa-miR-155 and CSNK1G2 mRNA in CLL suggests a regulatory effect on the IGVH-mutated subset. (a) Expression of CSNK1G2 mRNA in CLL patients (original cohort) subgrouped by high ( $n=11$, marked by dots in Supplementary Figure S1A) and low ( $n=11$, marked by asterisks in Supplementary Figure S1A) miR-155 expression. CSNK1G2 mRNA levels were quantified by quantitative reverse transcriptase-PCR and normalized to GUSB. The average CSNK1G2 mRNA expression in the high-miR-155 subgroup is arbitrarily set as one. The average CSNK1G2 mRNA expression in the low miR-155 subgroup is 1.62 times that of the high miR-155 subgroup ( $P<0.05)$. (b) CSNK1G2 mRNA levels are plotted against abundance of miR-155 in CLL/SLL B cells of 45 patients in the validation cohort. There was a weak but significant correlation $(P=0.027)$ between miR-155 and CSNK1G2 mRNA levels in these 45 CLLs. (c) Comparison of miR-155 and CSNK1G2 expression levels between the subgroup of 6 CLL patients with the highest miR-155 levels $(n=6$, high 6) and the subgroup of 6 CLL patients with the lowest miR-155 levels $(n=6$, low 6$)$. MiR-155 expression of the high subgroup was $\sim 9$ times that of the low $(P<0.0001)$. The miR-155 low subgroup showed slightly higher CSNK1G2 expression. However, statistical significance was not reached. (d and e) Correlation plots of miR-155 and CSNK1G2 expression levels for the IGVH-unmutated and -mutated CLL subsets are shown. Although there is no significant correlation between miR-155 and CSNK1G2 expression in the IGVH-unmutated CLLs, there is moderate negative correlation ( $r=-0.57$, $P=0.0048$ ) in the IGVH-mutated subset. (f and g). MiR-155 and CSNK1G2 mRNA levels of the top 6 (high 6) and bottom 6 (low 6 ) miR-155 expression in the IGVH-unmutated and -mutated CLL subgroups are indicated. Although there was no significance difference in the CSNK1G2 mRNA expression in the top and bottom six miR-155 expression within the IGVH-unmutated group, CSNK1G2 mRNA expression in the miR-155 low group is significantly higher $(P=0.04)$ within the $I G V H$-mutated group. 


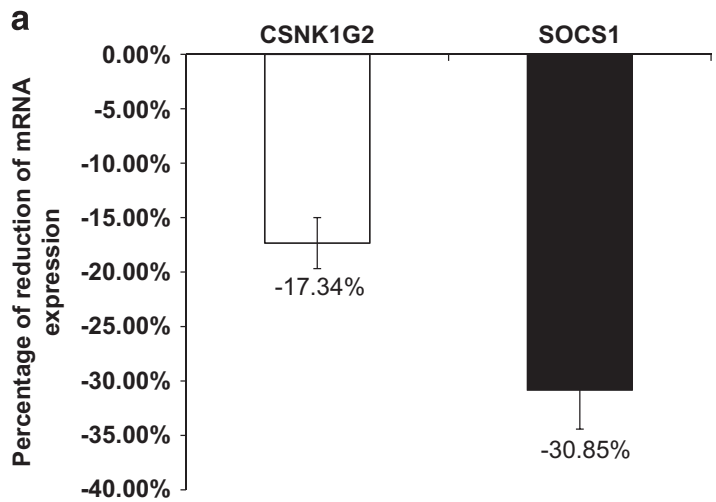

a b

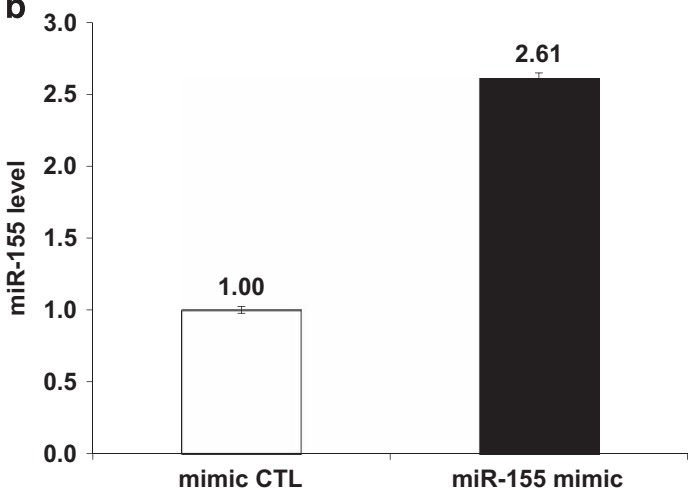

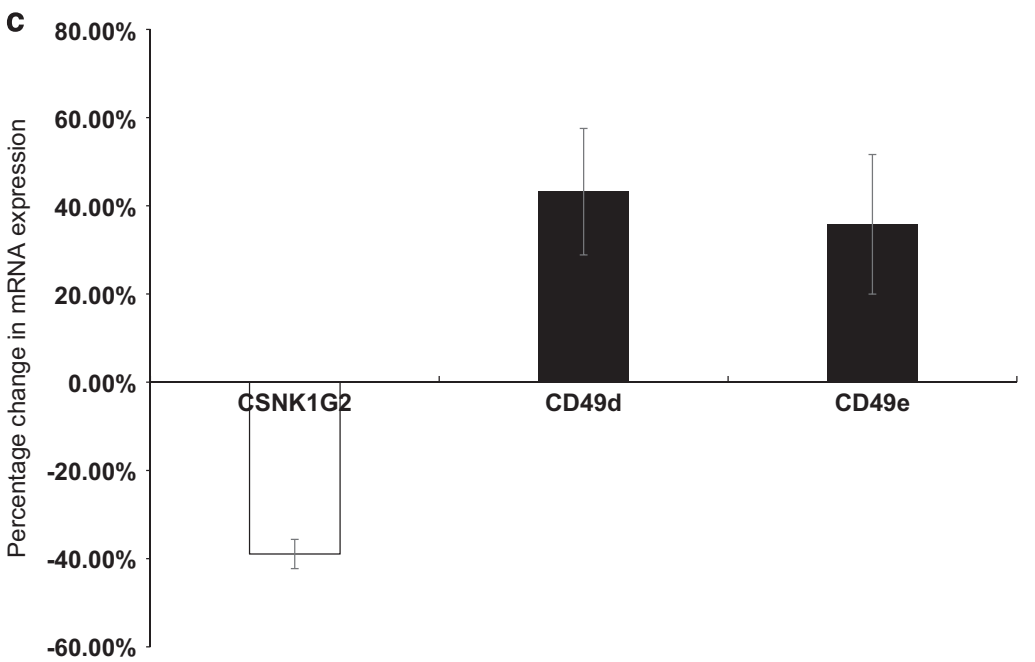

Figure 2. Enforced expression of miR-155 leads to a reduction in CSNK1G2 mRNA and CSNK1G2 knockdown results in increase of CD49d and CD49e expression levels in CLL cells. ( $\mathbf{a}$ and $\mathbf{b}$ ) Overexpression of miR-155 mimic in primary peripheral blood B cells of three IGVH-mutated CLL patients is accompanied by a decrease in CSNK1G2 mRNA level by $17.3+2.3 \%$ compared with the control (mimic CTL). The mRNA expression of SOCS1, a known target of miR-155, is decreased by $30.9 \pm 3.6 \%$ (a). The average level of miR-155 upon overexpression of miR-155 mimic is 2.6 times that of the control (b). Mimic CTL, miRCURY LNA microRNA mimic Negative Control 4 (Exiqon). (c) Purified peripheral blood B cells from five IGVH-mutated CLL patients ware transfected with siRNA against CSNK1G2. The average reduction of CSNK1G2 mRNA is 38.9\%. This decrease in CSNK1G2 is accompanied by an increase in CD49d and CD49e mRNA expression levels.

sphingomyelin synthesis. ${ }^{8}$ CSNK1G2 may regulate apoptosis in CLL cells through phosphorylation of CERT and regulation of sphingolipid metabolism. ${ }^{9}$

Recently, Teng et al. ${ }^{10}$ reported that miR-155 mimic enhanced both mRNA and protein levels of CD49e in A549 and HBE16 lung epithelial cells while the inhibitor suppressed protein expression. Interestingly, similar to miR-155, CD49d has been implicated as a poor prognostic marker for CLL. ${ }^{11,12}$ To determine whether CSNK1G2 may regulate CD49d and/or CD49e levels, we proceeded to knock down CSNK1G2 in CLL cells and see if this might lead to alteration in the levels of CD49d and CD49e. Indeed, when CSNK1G2 mRNA was reduced by $38.9 \%$ using siRNA, the levels of CD49d and CD49e transcripts were increased by $43.2 \%$ and $35.8 \%$, respectively (Figure $2 \mathrm{c}$ ). These results suggest a link between miR-155, CSNK1G2 and CD49d/e expression levels. It is conceivable that the positive modulatory effect of miR-155 on CD49d/e expression through downregulation of CSNK1G2 may be one of the underlying mechanisms accounting for the association of high levels of miR-155 with less favorable clinical outcome.

We have also identified two putative miR-155 gene targets in CLL in our differential microarray screening: ZNF652 and KLF3. Both of them harbor conserved miR-155-binding sites as predicted by Targetscan. Their interaction with miR-155 is also suggested by either luciferase reporter assay and/or quantitative reverse transcriptase-PCR results (data not shown). ZNF652 is a zinc finger protein that interacts with a ETO family protein CBFA2T3, a putative tumor suppressor in breast oncogenesis, to repress transcription. ${ }^{13}$ KLF3 is a member of the Kruppel-like factor (KLF) family of transcription factors that have diverse roles in cell proliferation, differentiation and apoptosis. Interestingly, KLF3 has been implicated to have important roles in B-cell biology. ${ }^{14} \mathrm{KLF} 3$ expression is high in resting quiescent $B$ cells but is reduced upon activation by $B$-cell receptor signaling, suggesting a role in maintaining cellular quiescence in B cells. KLF3 also appears to drive marginal zone B-cell differentiation. KLF3 is also the target gene in a common retroviral insertion site in B-cell lymphomas in mice, raising the possibility that alterations in KLF3 expression may promote B-cell lymphomagenesis. ${ }^{15}$

In summary, through global transcriptome profiling of CLL with differential miR-155 expression levels, we have successfully identified several putative targets of miR-155 in CLL. We have also performed additional studies to validate CSNK1G2 as a miR-155 target in CLL and proposed a mechanism of how this interaction may account for the association of high miR-155 with clinical aggressiveness of CLL. Our study highlights the feasibility of this strategy in identifying potential gene targets of deregulated miRNAs in the natural context of various types of hematological malignancies. The biological functions of CSNK1G2 and other putative targets of miR-155 (for example, KLF3) identified in this study may suggest a possible pathogenic role 
of these genes in CLL; additional studies to further investigate their contribution to CLL pathobiology may be warranted.

\section{CONFLICT OF INTEREST}

The authors declare no conflict of interest.

\section{ACKNOWLEDGEMENTS}

The work is supported by the Prince Family Foundation from Weill Cornell CLL Research Center.

T Zhang ${ }^{1,6}$, JK Davidson-Moncada ${ }^{1,2,6}$, P Mukherjee ${ }^{3}$, RR Furman ${ }^{4}$, E Bhavsar ${ }^{4}$, Z Chen $^{5}$, P Hakimpour ${ }^{2}$, N Papavasiliou ${ }^{2}$ and W Tam ${ }^{1}$

${ }^{1}$ Division of Hematopathology, Department of Pathology and

Laboratory Medicine, Weill Cornell Medicine, New York, NY, USA;

${ }^{2}$ Laboratory of Lymphocyte Biology, The Rockefeller University,

New York, NY, USA;

${ }^{3}$ Epigenomics Core Facility, Weill Cornell Medicine,

New York, NY, USA;

${ }^{4}$ Division of Hematology and Oncology, Department of Medicine, Weill Cornell Medicine, New York, NY, USA and

${ }^{5}$ Department of Healthcare Policy \& Research, Weill Cornell Medicine,

New York, NY, USA

E-mail: wtam@med.cornell.edu

${ }^{6}$ These authors contributed equally to the work.

\section{REFERENCES}

1 Davidson-Moncada J, Papavasiliou FN, Tam W. MicroRNAs of the immune system: roles in inflammation and cancer. Ann NY Acad Sci 2010; 1183: 183-194.

2 Due H, Svendsen P, Bodker JS, Schmitz A, Bogsted M, Johnsen HE et al. miR-155 as a biomarker in B-cell malignancies. Biomed Res Int 2016; 2016: 9513037.

3 Calin GA, Ferracin M, Cimmino A, Di Leva G, Shimizu M, Wojcik SE et al. A microRNA signature associated with prognosis and progression in chronic lymphocytic leukemia. N Engl J Med 2005; 353: 1793-1801.

4 Ferrajoli A, Shanafelt TD, Ivan C, Shimizu M, Rabe KG, Nouraee N et al. Prognostic value of miR-155 in individuals with monoclonal B-cell lymphocytosis and patients with B chronic lymphocytic leukemia. Blood 2013; 122: 1891-1899.

5 Cui B, Chen L, Zhang S, Mraz M, Fecteau JF, Yu J et al. MicroRNA-155 influences B-cell receptor signaling and associates with aggressive disease in chronic lymphocytic leukemia. Blood 2014; 124: 546-554.

6 Rai D, Karanti S, Jung I, Dahia PL, Aguiar RC. Coordinated expression of microRNA-155 and predicted target genes in diffuse large B-cell lymphoma. Cancer Genet Cytogenet 2008; 181: 8-15.

7 Meier J, Hovestadt V, Zapatka M, Pscherer A, Lichter P, Seiffert M. Genome-wide identification of translationally inhibited and degraded miR-155 targets using RNA-interacting protein-IP. RNA Biol 2013; 10: 1018-1029.

8 Hanada K, Kumagai K, Tomishige N, Yamaji T. CERT-mediated trafficking of ceramide. Biochim Biophys Acta 2009; 1791: 684-691.

9 Schwamb J, Feldhaus V, Baumann M, Patz M, Brodesser S, Brinker R et al. B-cell receptor triggers drug sensitivity of primary CLL cells by controlling glucosylation of ceramides. Blood 2012; 120: 3978-3985.

10 Teng Y, Miao J, Shen X, Yang X, Wang X, Ren L et al. The modulation of MiR-155 and MiR-23a manipulates Klebsiella pneumoniae adhesion on human pulmonary epithelial cells via integrin alpha5beta1 signaling. Sci Rep 2016; 6: 31918.

11 Bulian P, Shanafelt TD, Fegan C, Zucchetto A, Cro L, Nuckel H et al. CD49d is the strongest flow cytometry-based predictor of overall survival in chronic lymphocytic leukemia. J Clin Oncol 2014; 32: 897-904.

12 Majid A, Lin T, Best G, Fishlock K, Hewamana S, Pratt G et al. CD49d is an independent prognostic marker that is associated with CXCR4 expression in CLL. Leuk Res 2011; 35: 750-756.

13 Kumar R, Manning J, Spendlove HE, Kremmidiotis G, McKirdy R, Lee J et al. ZNF652, a novel zinc finger protein, interacts with the putative breast tumor suppressor CBFA2T3 to repress transcription. Mol Cancer Res 2006; 4: 655-665.

14 Pearson RC, Funnell AP, Crossley M. The mammalian zinc finger transcription factor Krüppel-like factor 3 (KLF3/BKLF). IUBMB Life 2011; 63: 86-93.

15 Warming S, Liu P, Suzuki T, Akagi K, Lindtner S, Pavlakis GN et al. Evi3, a common retroviral integration site in murine B-cell lymphoma, encodes an EBFAZ-related Krüppel-like zinc finger protein. Blood 2003; 101: 1934-1940.

\section{cc) (i)} International License. The images or other third party material in this article are included in the article's Creative Commons license, unless indicated otherwise in the credit line; if the material is not included under the Creative Commons license, users will need to obtain permission from the license holder to reproduce the material. To view a copy of this license, visit http://creativecommons.org/licenses/ by/4.0/

(c) The Author(s) 2017

Supplementary Information accompanies this paper on Blood Cancer Journal website (http://www.nature.com/bcj) 